\title{
Cinza da lenha para aplicação em cerâmica vermelha Parte I: características da cinza
}

\author{
(Firewood ash for application in red ceramic \\ Part I: characteristics of the ash)
}

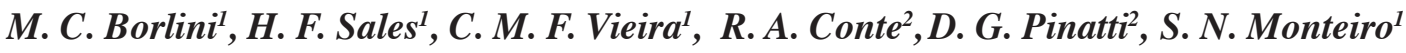 \\ ${ }^{1}$ Laboratório de Materiais Avançados - LAMAV, Centro de Ciências e Tecnologia - CCT \\ Universidade Estadual do Norte Fluminense - UENF \\ Av. Alberto Lamego, 2000, Pq Califórnia, Campos dos Goytacazes, RJ 28013-600 \\ ${ }^{2}$ Faculdade de Engenharia Química de Lorena FAENQUIL-DEMAR \\ C.P. 116, Lorena, SP 12600-000 \\ mborlini@terra.com.br
}

\begin{abstract}
Resumo
A lenha de eucalipto constitui-se no principal combustível utilizado pelas indústrias de cerâmica vermelha do município de Campos dos Goytacazes, Estado do Rio de Janeiro. Em princípio, esta lenha tem características promissoras para adição em cerâmica. Assim, esse trabalho tem por objetivo caracterizar a cinza proveniente da combustão de lenha predominantemente de eucalipto visando a incorporação na massa de cerâmica vermelha processada em indústrias de Campos dos Goytacazes. Foram realizados ensaios de fluorescência de raios X, difração de raios X, distribuição de tamanho de partícula, análise térmica (ATD/TG), porosimetria de mercúrio e microscopia eletrônica de varredura. Os resultados mostraram que a cinza da lenha se apresenta como aglomerados de partículas, sendo constituída principalmente por $\mathrm{Ca}, \mathrm{Si}, \mathrm{Mg}, \mathrm{K}$ e S.
\end{abstract}

Palavras-chave: cinza, lenha, cerâmica vermelha, caracterização.

Abstract

The eucalyptus firewood is one of the main fuels used by the industries of red ceramic in the municipal area of Campos of Goytacazes, State of Rio de Janeiro. In principle this firewood has promising characteristics for addition into ceramics. Thus, the present work has for objective to characterize the ash generated from burning firewood aiming at its incorporation into red ceramic products. The ash was submitted to X-ray diffraction, chemical composition, particle size distribution, thermal analysis (ATD/TG), mercury porosimetry and scanning electron microscopy tests. The results showed that the ash of the firewood comes as agglomerates of particles, being constituted mainly by $\mathrm{Ca}, \mathrm{Si}, \mathrm{Mg}, \mathrm{K}$ and $\mathrm{S}$.

Keywords: ash, firewood, red ceramics, characterization.

\section{INTRODUÇÃO}

No município de Campos dos Goytacazes, situado no norte do Estado de Rio de Janeiro, há uma área de aproximadamente $900 \mathrm{~km}^{2}$ com abundância em argilas aluviais formadas por sedimentos quaternários levados pelo Rio Paraíba. O setor de cerâmica vermelha inclui mais de 100 indústrias de tamanhos diferentes e com uma produção, principalmente de tijolos, estimada em 60 milhões de peças/mês. O combustível mais utilizado pelas indústrias de cerâmica vermelha é a lenha. Com um consumo médio 0,4 $\mathrm{m}^{3} /$ milheiro, densidade de $0,4 \mathrm{t} / \mathrm{m}^{3}$ e teor de cinzas da ordem de $3 \%$, estima-se uma geração mensal de aproximadamente 300 t de cinzas. O tipo de lenha mais comumente utilizado pelas indústrias cerâmicas de Campos dos Goytacazes -
RJ é proveniente do eucalipto. Em decorrência do uso da lenha, as indústrias cerâmicas tornam-se também grandes geradoras de cinzas.

Cinzas constituem um tipo de resíduo, contendo inclusive metais, que pode causar poluição do ar e ser responsável por graves problemas respiratórios na população atingida. Uma alternativa tecnológica para reduzir o impacto ambiental causado pela liberação indiscriminada de resíduos, particularmente as cinzas, seria a sua incorporação em produtos cerâmicos argilosos. Durante a fase de queima do processo de produção de cerâmica, a eliminação de metais potencialmente tóxicos pode ser promovida por volatilização, mudanças químicas e estabilização na fase vítrea, formada pela participação de aluminosilicatos e fundentes [1-4]. Além disso, o setor de cerâmica vermelha não requer 
controle rigoroso das matérias-primas, sendo mundialmente utilizado para incorporar resíduos industriais.

A nível mundial há um crescimento acentuado de trabalhos sobre cerâmicas obtidas com adições de resíduos sólidos, incluindo cinzas [5-10]. As motivações deste crescimento são encontrar formas estáveis para disposição final das cinzas visando evitar seus efeitos poluentes ao meio ambiente. Algumas classes de tecnologias têm sido estudadas, como a obtenção de vitrocerâmicas a partir de cinzas puras [10-12] e a adição de qualquer resíduo na composição de massa de cerâmica tradicional [5-7, 13-15]. Em alguns casos, não se objetiva obter produtos de maior valor agregado, mas simplesmente estabilizar os poluentes contidos nas cinzas.

O presente trabalho teve por objetivo caracterizar a cinza proveniente da combustão de lenha predominantemente de eucalipto visando sua incorporação na massa de cerâmica vermelha. Foram realizados ensaios de caracterização mineralógica, química, física e microestrutural. Na Parte II desse trabalho será apresentado um estudo sobre o efeito deste tipo de cinza no processamento e propriedades de uma cerâmica vermelha.

\section{MATERIAIS E MÉTODOS}

Nesse trabalho foi utilizada uma cinza proveniente da queima de lenha predominantemente de eucalipto pelas indústrias de cerâmica vermelha do município de Campos dos Goytacazes-RJ. Esta cinza foi caracterizada em termos de composição química e mineralógica, distribuição de tamanho de partícula, análise térmica (ATD/TG), porosimetria de mercúrio e microscopia eletrônica de varredura.

Acomposição química foi determinada por espectrometria de fluorescência de raios X (Philips PW 2400). A identificação das fases das cinzas foi realizada por difração de raios $\mathrm{X}$ (DRX). O DRX foi conduzido em amostras na forma de pó usando um difratômetro Seifert, modelo URD 65, operando com radiação $\mathrm{Cu}-\mathrm{K}_{\alpha}$ e $2 \theta$ de 5 a $70^{\circ}$.

A distribuição de tamanho de partícula foi realizada por peneiramento e sedimentação de acordo com a norma ABNT NBR-7181 [16].

A distribuição de tamanho de poros foi determinada por porosimetria de mercúrio usando um aparelho Pore Size 9310 da Micromeritics.

A caracterização microestrutural da cinza foi realizada através de microscopia eletrônica de varredura (MEV) utilizando um microscópio Zeiss, modelo DSM 960.

A análise térmica da cinza foi realizada no laboratório do Grupo de Preparação e Caracterização GPCM/DFA/ IFGW da Universidade Estadual de Campinas. As análises termogravimétrica (TG) e termodiferencial (DTA) foram conduzidas simultaneamente em um instrumento NETZSCH modelo STA 409 EP, operando sob fluxo de ar e taxa de aquecimento de $10{ }^{\circ} \mathrm{C} / \mathrm{min}$ até a temperatura máxima de $1200{ }^{\circ} \mathrm{C}$.

\section{RESULTADOS E DISCUSSÃO}

\section{Composição química e mineralógica}

A Tabela I mostra a composição química da cinza investigada. A composição química é caracterizada basicamente por alta quantidade de $\mathrm{CaO}$ e $\mathrm{SiO}_{2}$ e com quantidades significativas de $\mathrm{K}_{2} \mathrm{O}$ e $\mathrm{MgO}$. A quantidade elevada de $\mathrm{CaO}$ está relacionada a carbonato e hidróxido de cálcio enquanto o $\mathrm{SiO}_{2}$ está associado com a fase cristalina quartzo. A alta porcentagem de perda ao fogo $(21,5 \% \mathrm{em}$ peso) indica uma elevada fração de argilominerais. O teor

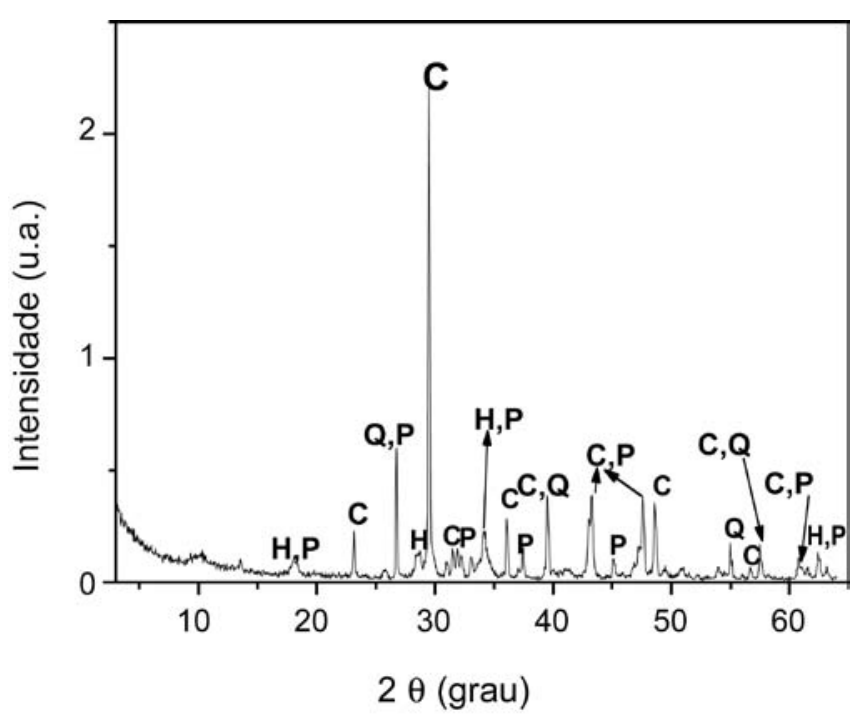

Figura 1: Difratograma de raios $\mathrm{X}$ da cinza da lenha. $\mathrm{C}=$ carbonato de cálcio, $\mathrm{H}=$ hidróxido de cálcio, $\mathrm{P}=$ fosfato de cálcio, $\mathrm{Q}=$ quartzo. [Figure 1: X-ray diffraction patterns of the ash of firewood. $C=$ calcium carbonate, $H=$ calcium hydroxide, $P=$ calcium phosphate, $Q=$ quartz.]

Tabela I - Composição química da cinza da lenha (\% peso).

[Table I - Chemical composition of the ash of firewood (wt. \%).]

\begin{tabular}{ccccccccccccc}
\hline $\mathrm{SiO}_{2}$ & $\mathrm{Al}_{2} \mathrm{O}_{3}$ & $\mathrm{Fe}_{2} \mathrm{O}_{3}$ & $\mathrm{~K}_{2} \mathrm{O}$ & $\mathrm{Na}_{2} \mathrm{O}$ & $\mathrm{MgO}$ & $\mathrm{CaO}$ & $\mathrm{MnO}_{2}$ & $\mathrm{Cl}$ & $\mathrm{SO}_{3}$ & $\mathrm{P}_{2} \mathrm{O}_{5}$ & $\mathrm{SrO}$ & $\mathrm{PF}$ \\
16,9 & 2,7 & 0,8 & 7,0 & 2,4 & 7,2 & 32,6 & 0,39 & 0,15 & 4,4 & 3,7 & 0,27 & 21,5 \\
\hline
\end{tabular}


relativamente elevado de $\mathrm{K}_{2} \mathrm{O}+\mathrm{Na}_{2} \mathrm{O}$ mostra que a cinza pode agir como um fundente potencial e pode contribuir para melhorar as propriedades de queima através da redução da porosidade pela formação de fase líquida em reação com $\mathrm{SiO}_{2}$ e $\mathrm{Al}_{2} \mathrm{O}_{3}$. $\mathrm{O} \mathrm{MgO}$ também pode atuar como fundente durante a etapa de queima. A cinza apresenta ainda traços de Ti, Ni, Rb.

A Fig. 1 apresenta os resultados da análise por DRX da cinza. Analisando os picos do difratograma verificase que a principal fase cristalina existente na cinza é o carbonato de cálcio confirmando os resultados da Tabela 1. Observa-se ainda a presença de quartzo, hidróxido e fosfato de cálcio.

\section{Morfologia e granulometria}

A Fig. 2 apresenta as micrografias da cinza. Observase que esta cinza é formada por aglomerados com uma forma predominantemente arredondada resultante da aglomeração de partículas. Ou seja, a cinza não se apresenta como partículas separadas, mas sim, como um produto sinterizado e bastante poroso em sua estrutura.

A Fig. 3 apresenta a distribuição granulométrica da cinza. Observa-se que esta apresenta tamanho de aglomerados compreendidos entre 2 e $0,01 \mathrm{~mm}$. O tamanho médio observado é de $0,15 \mathrm{~mm}$. A técnica empregada pode não ter sido adequada para determinar o tamanho real das partículas, sendo possível identificar apenas os aglomerados.

A Fig. 4 mostra a distribuição do tamanho dos poros da cinza. Pode ser observado que o diâmetro dos poros está na faixa de 0,0072 a 107,77 $\mu \mathrm{m}$, sendo predominante o tamanho de poro de $0,9716 \mu \mathrm{m}$. A cinza apresenta uma densidade, medida por porosimetria de mercúrio, de $2,038 \mathrm{~g} / \mathrm{cm}^{3}$ e uma porosidade aberta de 48,95\%.
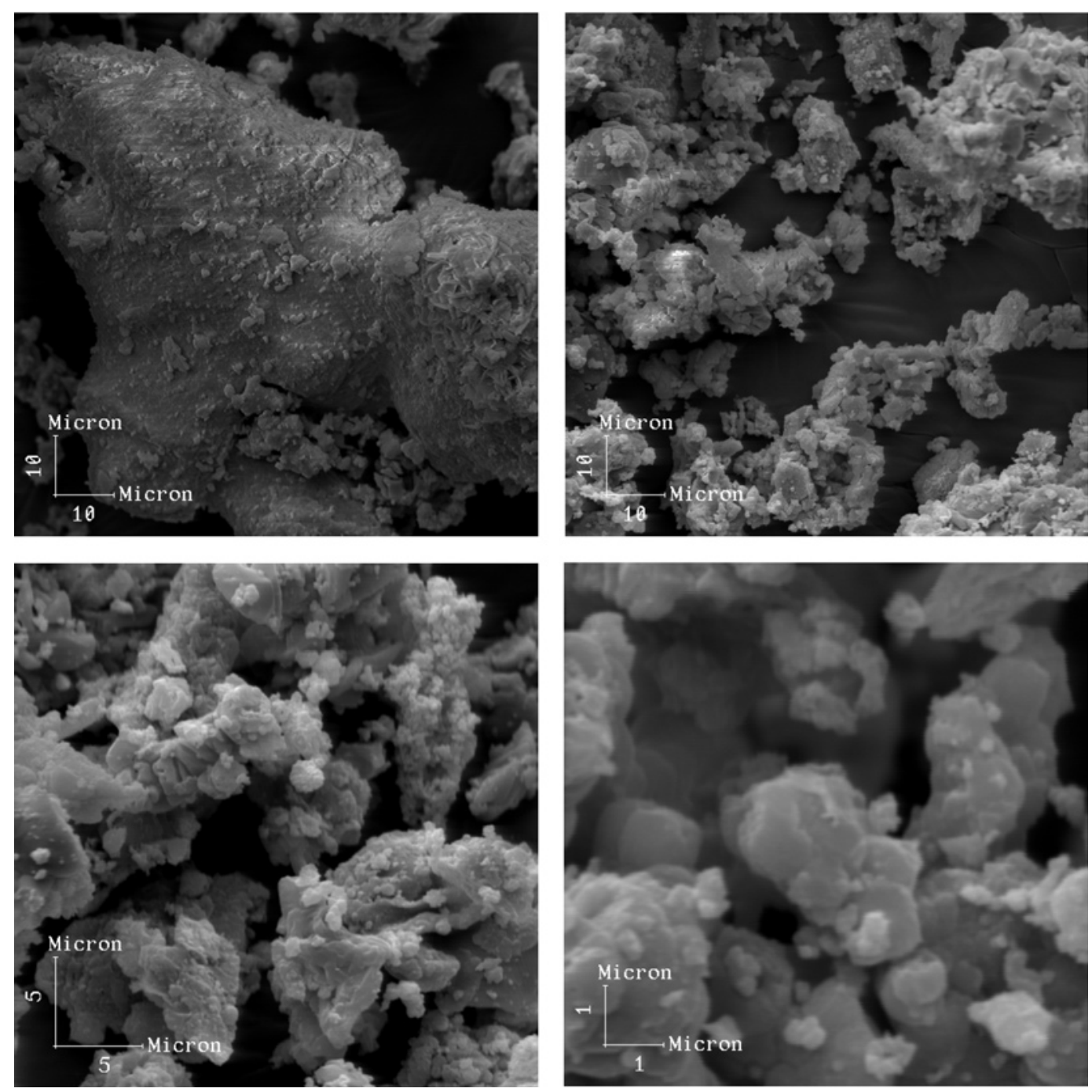

Figura 2: Micrografias da cinza da lenha obtidas por microscopia eletrônica de varredura.

[Figure 2: Microstructure of the ash of firewood obtained by scanning electron microscopy.] 


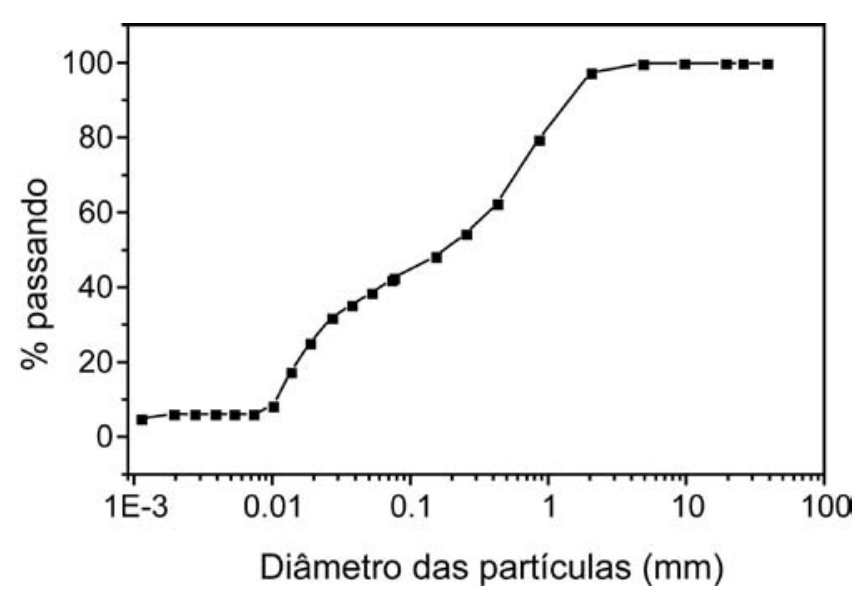

Figura 3: Distribuição Granulométrica da cinza da lenha. [Figure 3: Particle size distribution of the ash of firewood.]

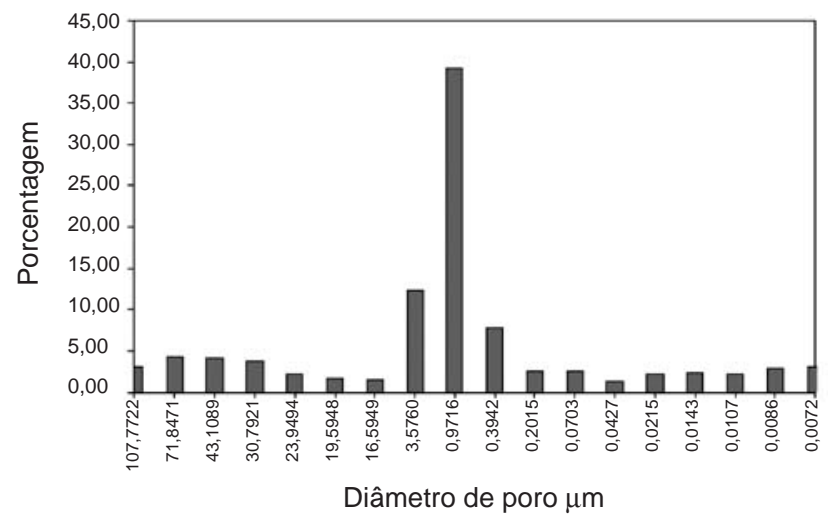

Figura 4: Distribuição de tamanho de poro da cinza da lenha [Figure 4: Pore size distribution of the ash of firewood.]

A área superficial é de $26,34 \mathrm{~m}^{2} / \mathrm{g}$. Esta porosidade relativamente alta da cinza pode facilitar a etapa de secagem das cerâmicas.

\section{Comportamento térmico}

O comportamento térmico da cinza foi estudado por meio de análises de termogravimetria (TG) e termodiferencial (DTA). A Fig. 5 mostra as curvas de TG e DTA da cinza da lenha. Os aspectos fundamentais do comportamento térmico das cinzas são:

(A) pico endotérmico a $183{ }^{\circ} \mathrm{C}$ devido à perda de água adsorvida;

(B) pico endotérmico a $466{ }^{\circ} \mathrm{C}$ devido à desidratação de sulfato de magnésio $\left(\mathrm{MgSO}_{4} \cdot \mathrm{nH}_{2} \mathrm{O}\right)$ [5];

(C) pico endotérmico em torno de $568{ }^{\circ} \mathrm{C}$ associado à transformação alotrópica do quartzo- $\alpha$ para quartzo- $\beta$;

(D) pico endotérmico a $828{ }^{\circ} \mathrm{C}$ devido à decomposição do carbonato de cálcio e associado a uma perda de peso de 15\%.

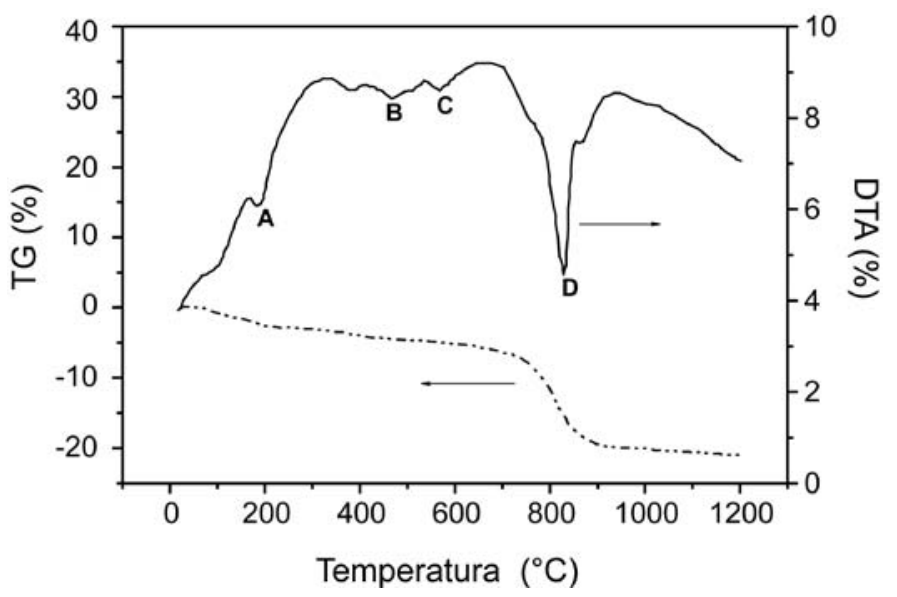

Figura 5: Curva termogravimétrica (TG) e termodiferencial (DTA) da cinza da lenha.

[Figure 5: Termogravimetry (TG) and termodiferencial (DTA) curves of the ash of firewood.]

\section{CONCLUSÕES}

Acinza obtida da combustão de lenha predominantemente de eucalipto em fornos para produção de cerâmica em Campos dos Goytacazes, exibe as seguintes características:

- é especialmente rica em cálcio, sendo composta predominantemente por carbonato de cálcio;

- apresenta aglomerados de partículas predominantemente esféricos e porosos e com tamanho médio de $0,15 \mathrm{~mm}$.

- quando a cinza for adicionada em argila, o teor relativamente elevado de óxidos alcalinos e alcalino-terrosos pode contribuir para redução da porosidade de queima através da formação de fase líquida.

\section{AGRADECIMENTOS}

Os autores agradecem à COPPE/UFRJ pela utilização do microscópio eletrônico de varredura, a GPCM/DFA/ IFGW-UNICAMP pela realização da análise térmica e a FAPERJ (protocolo $\mathrm{n}^{\circ}$ 1700802004) pelo apoio ao trabalho desenvolvido. É também motivo de agradecimento o suporte parcial dado pela CAPES, CNPq e TECNORTE/ FENORTE.

\section{REFERÊNCIAS}

[1] M. C. Borlini, Dissertação de Mestrado, DEMAR, FAENQUIL (2002).

[2] CTCV - Centro Tecnológico da Cerâmica e do Vidro, Principais problemas Ambientais na Indústria Cerâmica, Portugal (1997).

[3] A. Blasco, A. Escardino, G. Busani, E. Monfort, J. L. Amorós, J. Enrique, V. Beltrán, P. Negre, AICE-ITC, Espanha (1992).

[4] M. Dondi, M. Marsigli, B. Fabbri, Tile \& Brick Int. 13, 3 (1997) 218. 
[5] M. Dondi, G. Ercolani, G. Guarini, M. Raimondo, J. Eur. Ceram. Soc. 22 (2002) 1729.

[6] M. Dondi, G. Guarini, M. Raimondo, I. Venturi, J. Eur. Ceram. Soc. 22 (2002) 1737.

[7] M. Dondi, G. Guarini, M. Raimondo, A. Ruffini, J. Eur. Ceram. Soc. 22 (2002) 1749.

[8] L. Barbieri, A. Corradi, I. Lancellotti, T. Manfredini, Waste Managementy 8, 22 (2002) 859.

[9] C. R. Cheeseman, S. M. Rocha, C. Sollars, S. Bethanis, A. R. Boccaccini, Waste Management 10, 23 (2003) 907.

[10] J. M. Kim, H. S. Kim, J. Eur. Ceram. Soc. 24 (2004) 2373.

[11] A. Karamanova, M. Pelinoa, A. Hreglichb, J. Eur. Ceram. Soc. 23 (2003) 827.
[12] A. Karamanov, M. Pelino, M. Salvo, I. Metekovits, J. Eur. Ceram. Soc. 23 (2003) 1609.

[13] C. T. Kniess, K. W. Milanez, N. C. Kuhnen, H. G. Riella, A. M. Segadães, Fourth International Latin-American Conference on Powder Technology, Guarujá, SP (2003) cdrom, ref. 17/17.

[14] I. S. Tavares, J. M. D. Soares, R. J. B. Pinheiro, Anais do $47^{\circ}$ Congresso Brasileiro de Cerâmica, J. Pessoa, Pb (2003) cdrom, ref. 5-40.

[15] L. M. H. Quintana, J. M. D. Soares, T. M. Pacheco, M. A. Aquere, T. Soares, Anais do $47^{\circ}$ Congresso Brasileiro de Cerâmica, J. Pessoa, Pb (2003) cdrom, ref. 5-50.

[16] ABNT, Solo - Análise Granulométrica, NBR - 7181 (1984) 13.

(Rec.12/11/2004, Ac.03/06/2005) 